\title{
Stability condition of a class of nonlinear feedback systems: reduction to a convex problem
}

\author{
AUTHOR(S):
}

Hagiwara, T; Miyake, Y; Furutani, E; Araki, M

\section{CITATION:}

Hagiwara, T ...[et al]. Stability condition of a class of nonlinear feedback systems:

reduction to a convex problem. IEEE Transactions on Automatic Control 1999, 44(8): 15731577

\section{ISSUE DATE:}

1999-08

URL:

http://hdl.handle.net/2433/39969

\section{RIGHT:}

(c) 1999 IEEE. Personal use of this material is permitted. However, permission to reprint/republish this material for advertising or promotional purposes or for creating new collective works for resale or redistribution to servers or lists, or to reuse any copyrighted component of this work in other works must be obtained from the IEEE. 


\section{Stability Condition of a Class of Nonlinear Feedback Systems: Reduction to a Convex Problem}

Tomomichi Hagiwara, Yoshikazu Miyake, Eiko Furutani, and Mituhiko Araki

\begin{abstract}
This paper gives a new criterion for input-output stability of a class of nonlinear feedback systems. Roughly speaking, it is most useful in such a practical situation where the nonlinearity in the system is "almost time-invariant and memoryless" but with "slight time-variations and dynamics." It involves two free parameters and contains the circle criterion and the Popov criterion as special cases. In fact, it extends these two famous criteria in such a way that the conservatism of the circle criterion can be reduced when the time-variations and dynamics of the nonlinearity are "relatively small." It is also shown that the existence of the free parameters that fulfill the stability condition can be checked exactly, by reducing it to a convex problem in the frequency domain.
\end{abstract}

Index Terms - Circle criterion, convex optimization, input-output stability, Popov criterion.

\section{INTRODUCTION}

It is widely accepted that virtually all real systems are more or less nonlinear; for example, the plant may be nonlinear because of its backlash characteristics, or the controller may be nonlinear because of the saturation of the actuator output. Since stability is the most fundamental property that is required of a control system, it is extremely important to have a sharp (or less conservative) stability condition for nonlinear systems.

It is often possible to separate a nonlinear system into a linear part and a nonlinear part, which are connected with each other as in Fig. 1. In many practical situations, the nonlinear part belongs to the class of so-called sector nonlinearities (see Section II for details), and two types of stability conditions for such nonlinearities are well known: the circle criterion and the Popov criterion [2], [5]. The circle criterion can be applied to a general sector nonlinearity with memory (dynamics) and time-varying characteristics, while the Popov criterion can only deal with a memoryless time-invariant sector nonlinearity. The price is that the condition the circle criterion requires is much stronger than that required by the Popov criterion.

Recently, a more general stability condition was derived that includes the above two famous existing conditions as special cases [3], [4] and was referred to as the shifted Popov criterion. Roughly speaking, the feature of the new criterion is that it can give a weaker condition for stability than the circle criterion does when the nonlinear part belongs to a certain class of time-varying (or state-dependent) sector nonlinearities (see Section II for the details of the class). To consider such a class is natural and the criterion is powerful in many practical situations where the nonlinear characteristics are almost time-invariant but change slightly with time.

Now, we are in a position to state the purpose of this paper. To put it briefly, the aim is to develop the parallel results to the shifted Popov criterion under the setting of input-output stability; Lyapunov stability was studied in the former work [3], [4]. There are three motivations for such a research direction. First, it enables us to deal with an infinite-dimensional system. Namely, we can consider,

Manuscript received March 20, 1997. Recommended by Associate Editor, G. M. Huang.

The authors are with the Department of Electrical Engineering, Kyoto University, Yoshida, Sakyo-ku, Kyoto 606-01, Japan.

Publisher Item Identifier S 0018-9286(99)05456-2.

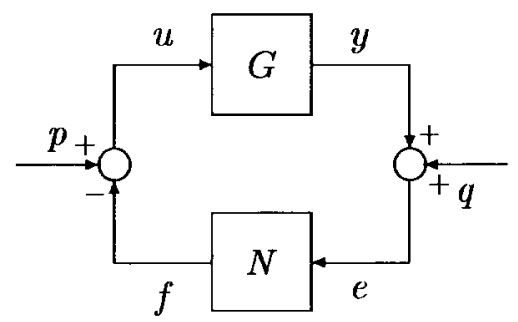

Fig. 1. Nonlinear feedback system $\Sigma_{a}$.

for example, a system with a pure delay as the linear part of the nonlinear feedback system. Second, a (stable) dynamical nonlinearity can also be dealt with by the results of this paper, which should be useful in robust stability analysis. The third motivation is more or less pedagogical. Namely, by taking the input-output viewpoint, we can reach the condition for stability fairly easily in a transparent manner. The derived condition is the same as that obtained by the Lyapunov stability setting except for some minor technical details, whereas the derivation in that setting is more involved and less intuitive. Our derivation in the input-output setting makes use of scaling among loops, which is reminiscent of the $\mu$ analysis or the $H_{\infty}$ problem with constant scaling [7]. The contribution of this paper, however, is not limited to the extension to the input-output setting; this paper has a significant advance over the previous work in having shown the following: to check if the stability condition is satisfied can be reduced to a convex problem, which can be solved easily [1].

The notation used in this paper is standard. $L_{2}$ denotes the space of (Lebesgue) square integrable functions over the nonnegative time interval, and $L_{2 e}$ denotes its extended space. For $f \in L_{2}$, the $L_{2}$ norm of $f$ is denoted by $\|f\|$, while for $f \in L_{2 e},\|f\|_{L_{2}[0, T]}$ is defined by

$$
\|f\|_{L_{2}[0, T]}:=\left(\int_{0}^{T}\|f(t)\|^{2} d t\right)^{1 / 2} .
$$

The $L_{2}$-induced norm of a system is denoted by $\|\cdot\|$ if it is bounded on $L_{2}$. Also, $\mathcal{A}$ is defined as the set of functions $f(\cdot)$ of the form

$$
f(t)=\sum_{i=0}^{\infty} f_{i} \delta\left(t-t_{i}\right)+f_{a}(t)
$$

where $\delta(\cdot)$ denotes the unit delta function, $0 \leq t_{0}<t_{1}<\cdots$ are constants, $f_{a}(t)=0$ for $t<0$, and

$$
\sum_{i=0}^{\infty}\left|f_{i}\right|<\infty, \quad \int_{0}^{\infty}\left|f_{a}(t)\right| d t<\infty .
$$

\section{Problem SetuP}

Let us consider the nonlinear feedback system $\Sigma_{a}$ shown in Fig. 1. Here, $G$ denotes the linear part of the system, and we assume that it is time-invariant. The nonlinear part of the system is denoted by $N$, and we assume that it can be modeled as the parallel connection of a memoryless time-invariant nonlinearity and a (possibly) dynamical time-varying nonlinearity as in Fig. 2. Namely, $f=N(e)$ satisfies

$$
f=f_{0}+f_{1}, \quad f_{i}=N_{i}(e) \quad(i=0,1)
$$




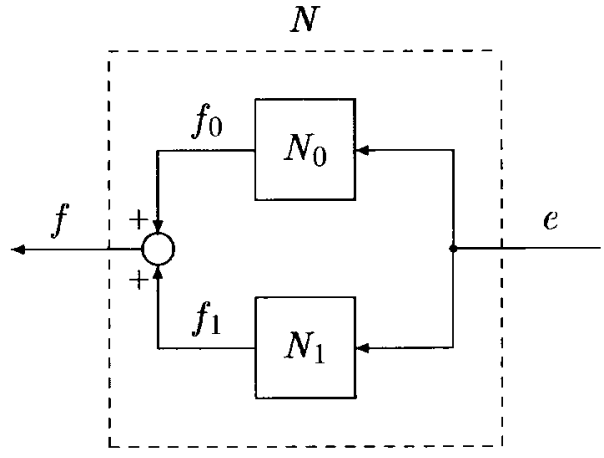

Fig. 2. Structure of the nonlinearity $N$.

where $N_{0}: L_{2 e} \rightarrow L_{2 e}$ is memoryless and time-invariant, while $N_{1}: L_{2 e} \rightarrow L_{2 e}$ is (possibly) dynamical and time-varying. We further assume that $N_{0}$ belongs to the sector $[a, b](a \leq b<\infty)$, and $N_{1}$ belongs to the sector $[-\varepsilon, \varepsilon](\varepsilon>0)$, where sectors are defined as follows.

Definition 1 [5]: The memoryless time-invariant nonlinearity $N_{0}$ is said to belong to the sector $[a, b]$ if there exists a nonlinear characteristic function $\nu_{0}(\cdot)$ such that

$$
a \leq \frac{\nu_{0}(e)}{e} \leq b(e \neq 0), \quad \nu_{0}(0)=0
$$

and $f_{0}(t)=\nu_{0}(e(t))$ for each time $t$.

Definition 2 [5]: The (possibly) dynamical and time-varying nonlinearity $N_{1}$ is said to belong to the sector $[a, b]$ if

$$
\left\|f_{1}-k e\right\|_{L_{2}[0, T]} \leq r\|e\|_{L_{2}[0, T]}, \quad \forall T \geq 0, \forall e \in L_{2 e}
$$

where

$$
k:=(b+a) / 2, \quad r:=(b-a) / 2 .
$$

Remark 1: Definition 2 is stated in a general form for later use. Since we assume that $N_{1}$ belongs to the symmetric sector $[-\varepsilon, \varepsilon]$ as mentioned above, we in fact assume that

$$
\left\|f_{1}\right\|_{L_{2}[0, T]} \leq \varepsilon\|e\|_{L_{2}[0, T]}, \quad \forall T \geq 0, \forall e \in L_{2 e} .
$$

The idea behind the above assumption is that many nonlinearities in practice are almost memoryless and time-invariant (as modeled by $N_{0}$ ) but with slight time variations and dynamics (as modeled by $\left.N_{1}\right)$. For example, suppose that $f(t)=\nu(t, e(t))$ is true for each $t$ for some (probably unknown) time-varying nonlinear characteristic function $\nu(t, \cdot)$, where the graph of $\nu(t, \cdot)$ for each fixed $t$ is known to lie within the shaded area of Fig. 3. Then, it is easy to see that we can choose ${ }^{1} a=0, b=1$, and $\varepsilon=0.2$ in the description of $N$ by $N_{0}$ and $N_{1}$. On the other hand, if we were to model this nonlinearity only with $N_{1}$, we would have to consider a much wider sector [0, 1.2], which will make the stability analysis conservative [3], [4]. In the following, we assume $a=0$ and hence $b \geq 0$ without loss of generality. Also, $N_{0}$ is called the primary part and $N_{1}$ the deviation part of the nonlinearity $N$.

Having described the setup of the system we deal with, the purpose of this paper is to give a condition that ensures the input-output stability of the nonlinear feedback system $\Sigma_{a}$.

Remark 2: In [3] and [4], a time-varying (or linear-part-state dependent) but memoryless nonlinearity satisfying

$$
\left\|f_{1}(t)\right\| \leq \varepsilon\|e(t)\|
$$

\footnotetext{
${ }^{1}$ To be more precise, we can actually choose $a=0.2, b=1$, and $\varepsilon=0.2$.
}

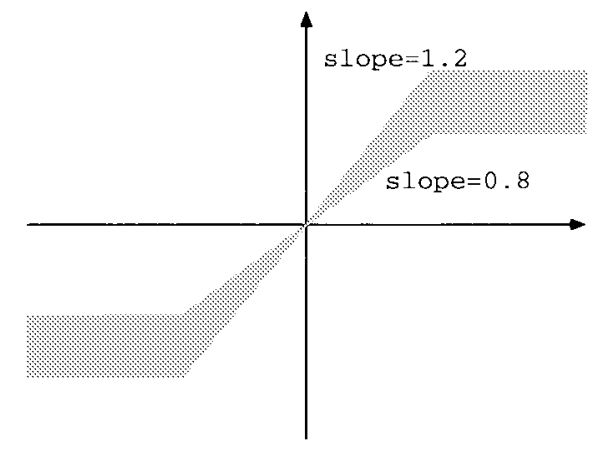

Fig. 3. An example of nonlinearity.

for each $t$ was considered as the deviation part $N_{1}$. Integrating the square of the above over the time interval $[0, T]$, we have

$$
\left\|f_{1}\right\|_{L_{2}[0, T]}^{2} \leq \varepsilon^{2}\|e\|_{L_{2}[0, T]}^{2}
$$

which is nothing but (8). This implies that the class of the deviation parts studied in [3] and [4] is a subset of the class we deal with here.

\section{MAIN RESUlT}

We use the following definition of input-output stability for the system $\Sigma_{a}$, which is the same stability notion as that used in the Popov criterion in the input-output setting.

Definition 3: $\Sigma_{a}$ is said to be pseudo $L_{2}$-stable if there exists some $\gamma$ such that

$$
\left\|\left[\begin{array}{l}
f \\
y
\end{array}\right]\right\| \leq \gamma\left\|\left[\begin{array}{l}
p \\
q \\
\dot{q}
\end{array}\right]\right\|\left(\text { whenever } p, q, \dot{q} \in L_{2}\right)
$$

where $\dot{q}$ denotes the derivative of $q$.

Then, we can obtain the following main result (see the Appendix for the proof).

Theorem 1: Suppose that $g, \dot{g} \in \mathcal{A}$, where $g$ is the impulse response of $G$ and $\dot{g}$ denotes its derivative. Let $\hat{G}(s)$ denote the transfer function of $G$. Then, the nonlinear feedback system $\Sigma_{a}$ is pseudo $L_{2}$-stable if there exist a real number $\theta$ and a positive number $\rho$ such that

$$
\bar{f}(\theta, \rho):=\sup _{\omega \in[0, \infty]} f_{\omega}(\theta, \rho)<0
$$

where

$$
\begin{aligned}
f_{\omega}(\theta, \rho)= & -(1+b \cdot \operatorname{Re}\{(1+j \omega \theta) \hat{G}(j \omega)\}) \\
& +\frac{b \varepsilon}{2 \rho}|(1+j \omega \theta) \hat{G}(j \omega)|^{2}+\left(\varepsilon^{2}+\frac{b \varepsilon \rho}{2}\right)|\hat{G}(j \omega)|^{2} .
\end{aligned}
$$

Remark 3: Except for some minor technical details, the condition (12) turns out to be essentially the same as that obtained in the Lyapunov stability setting [3], [4]. Nonetheless, the above result has an independent value, since it has some advantages over the previous work, as mentioned in Section I; it can be applied to, e.g., infinite-dimensional systems, too.

Now, when $N$ is memoryless and time-invariant, so that the deviation part $N_{1}$ vanishes (and hence $\varepsilon=0$ ), the above condition 
reduces to the Popov condition

$$
\inf _{\omega \in[0, \infty]}(b \cdot \operatorname{Re}\{(1+j \omega \theta) \hat{G}(j \omega)\})>-1 .
$$

On the other hand, when $N$ is "totally time-varying and/or dynamic," so that the "primary part" $N_{0}$ vanishes (and hence $b=0$ ), it reduces to the circle condition (or the small-gain condition)

$$
\sup _{\omega \in[0, \infty]}(\varepsilon \cdot|\hat{G}(j \omega)|)<1 \text {. }
$$

(By the loop transformation technique [5], we can also verify that it can represent the general form of the circle criterion as well.) This implies that the above condition includes the Popov criterion and the circle criterion as special cases and connects these two famous criteria "continuously." To quote the terminology used in [3] and [4], the above theorem is a "shifted Popov criterion," because we can conclude stability by drawing a "shifted Popov locus, ${ }^{2}$ " and checking that it lies to the right of the line passing through the point $-1+j 0$ and with slope $1 / \theta$. A difficulty in checking stability in this way is that the shifted locus is dependent on $\theta$ and $\rho$, and the line is also dependent on $\theta$ so that it is not obvious how to find appropriate values for these two parameters. Unfortunately, no systematic way for resolving this was given in [3] and [4], and hence stability was checked on a trial-and-error basis (to be precise, the parameters $\theta$ and $\psi$ were used there instead, where $\psi$ is equal to $\rho^{-1}$ in our notation). In contrast, here we can give a simple solution to this difficulty; checking the existence of the parameters that satisfy the condition (12) can be reduced to a convex problem.

To see this, note that

$$
\begin{aligned}
& {\left[\begin{array}{cc}
\partial^{2} f_{\omega} / \partial \theta^{2} & \partial^{2} f_{\omega} / \partial \theta \partial \rho \\
\partial^{2} f_{\omega} / \partial \theta \partial \rho & \partial^{2} f_{\omega} / \partial \rho^{2}
\end{array}\right]} \\
& \quad=\frac{b \varepsilon}{\rho^{3}}|\hat{G}(j \omega)|^{2}\left[\begin{array}{cc}
\rho^{2} \omega^{2} & -\rho \omega^{2} \theta \\
-\rho \omega^{2} \theta & 1+\omega^{2} \theta^{2}
\end{array}\right] \geq 0
\end{aligned}
$$

is true for all $\theta$ and $\rho>0$. Hence, $f_{\omega}(\theta, \rho)$ is convex for each fixed $\omega$, so that

$$
\bar{f}(\theta, \rho):=\sup _{\omega \in[0, \infty]} f_{\omega}(\theta, \rho)
$$

is convex, too. Therefore, existence of the parameters $\theta$ and $\rho$ that prove stability via Theorem 1 can be checked readily with such convex optimization algorithms as the cutting-plane algorithm and the ellipsoid method [1].

The advantage of this convexity approach is obvious. For example, for fixed $b$, we can readily determine, via the bisection search, the upper bound of $\varepsilon$ for which Theorem 1 can assure stability. Alternatively, the upper bound for $b$ can also be determined for fixed $\varepsilon$. Furthermore, for fixed $b$ and $\varepsilon$, we can determine the "pure-delay margin" of the system. Here, by "pure-delay margin," we mean the range of the extra pure delay $\Delta T_{L}$ (which could be negative) that the plant $G$ can accommodate (on the top of the nominal pure delay $T_{L 0}$ ) while stability of the feedback system is guaranteed. The calculation of this margin is based on the observation that $\Delta T_{L}$ affects only the first term on the right-hand side of (13) and is carried out with alternate iterations involving the modification of the function $\bar{f}(\theta, \rho)$

\footnotetext{
${ }^{2}$ This locus is obtained by drawing the Popov locus magnified by the factor of $b$ and shifting each point on the locus to the left by the amount of the second and third terms on the right-hand side of (13).
}

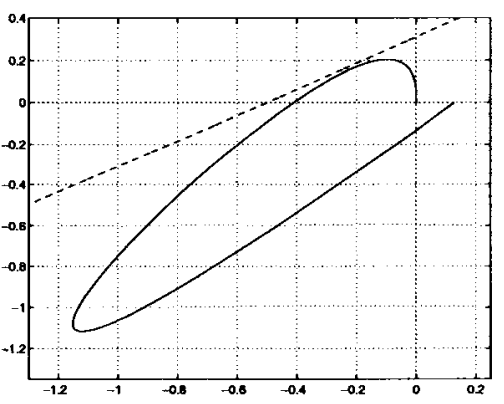

Fig. 4. Shifted Popov locus for $(\theta, \rho)=(1.61085,3.29146)$

and the optimization of that modified function. The details will be explained in the following section with a numerical example.

\section{EXAMPLE}

Let us consider the nonlinear feedback system of Fig. 1 with

$$
\hat{G}(s)=\frac{5}{(s+1)^{2}(s+2)} e^{-T_{L} s}
$$

where the nominal value of the pure delay $T_{L}$ is $T_{L 0}=0.05$, and the sizes of the sectors associated with the nonlinear element are given by $b=2$ and $\varepsilon=0.2$. The circle criterion applied to this system (with sector $[-\varepsilon, b+\varepsilon]=[-0.2,2.2]$ ) cannot assure stability of this system.

We applied the ellipsoid method to search for a point $(\theta, \rho)$ that satisfies the condition (12), taking the initial ellipsoid as the circle with radius 100 centered at $(0,1)$ (this value of $(\theta, \rho)$ corresponds to the circle criterion mentioned above). After 17 iterations, we reached the point $(\theta, \rho)=(1.61085,3.29146)$ such that (12) is satisfied. Namely, the feedback system considered here is assured to be pseudo $L_{2}$-stable. The associated shifted Popov locus is shown in Fig. 4; we can verify that the shifted Popov locus lies to the right of the critical line.

In general, to identify the pure delay $T_{L}$ of a system exactly is a hard task, and hence it is practically very important that stability is assured for some relatively wide range of $T_{L}$ around its nominal value $T_{L 0}$. As we already stated at the end of the preceding section, we can determine the range of $T_{L}$ for which we can assure stability. The method is illustrated below, using the same example. To make clear that the underlying time delay is $T_{L}$, the function $f_{\omega}(\theta, \rho)$ defined in (13) will be denoted by $f_{\omega}^{T_{L}}(\theta, \rho)$, and similarly, $\bar{f}(\theta, \rho)$ defined in (12) will be denoted by $\bar{f}^{T} L(\theta, \rho)$ in the following.

Continuing the iterations to search for the optimal point $(\theta, \rho)=\left(\theta_{1}, \rho_{1}\right)$ that minimizes the function $\bar{f}^{T_{L 0}}(\theta, \rho)$, we obtain $\left(\theta_{1}, \rho_{1}\right)=(0.868918,1.98249)$, for which we naturally have $\bar{f}^{T_{L 0}}\left(\theta_{1}, \rho_{1}\right)<0$. Noting that the change of $T_{L}$ affects only the first term on the right-hand side of (13), we can compute, for each $\omega$, the smallest positive $\overline{\Delta T}_{L}(\omega)$ such that $f_{\omega}^{T_{L}+\overline{\Delta T}_{L}(\omega)}\left(\theta_{1}, \rho_{1}\right)=0$, if it exists (see Fig. 5) - if no such $\overline{\Delta T}_{L}(\omega)$ exists, then define $\overline{\Delta T}_{L}(\omega)=\infty$. We now define

$$
T_{L 1}=T_{L 0}+\inf _{\omega \in[0, \infty]} \overline{\Delta T}_{L}(\omega)=0.145346
$$

Then, obviously, we have $\bar{f}^{T_{L 1}}\left(\theta_{1}, \rho_{1}\right)=0$. But, optimizing the function $\bar{f}^{T_{L 1}}(\theta, \rho)$, we obtain $\bar{f}^{T_{L 1}}\left(\theta_{2}, \rho_{2}\right)<0$ for $\left(\theta_{2}, \rho_{2}\right)=$ $(0.808146,1.72389)$. Then, using the same procedure as before,

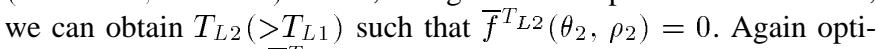
mizing the function $\bar{f}^{T_{L 2}}(\theta, \rho)$ and repeating the same procedures, 


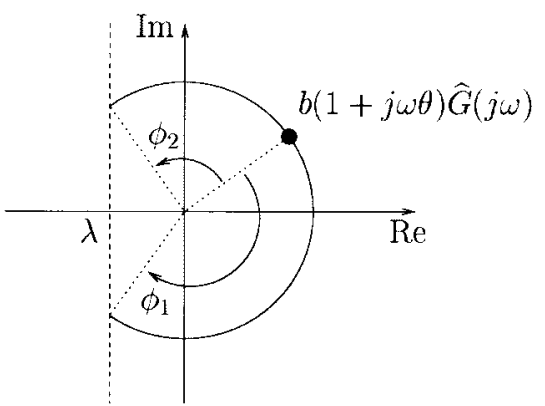

Fig. 5. Computation of $\overline{\Delta T}_{L}(\omega)$ and $\underline{\Delta T_{L}}(\omega) \quad(\lambda=-1+$ $(b \varepsilon / 2 \rho)|(1+j \omega \theta) \hat{G}(j \omega)|^{2}+\left(\varepsilon^{2}+(b \varepsilon \rho / 2)\right)|\hat{G}(j \omega)|^{2}, \overline{\Delta T}_{L}(\omega)=$ $\left.\phi_{1} / \omega, \Delta T_{L}(\omega)=-\phi_{2} / \omega\right)$.

we obtain the nondecreasing sequence $\left\{T_{L 0}, T_{L 1}, T_{L 2}, \cdots\right\}$, which converges to $\bar{T}_{L}=0.146877$. It is obvious that stability is assured for any $T_{L}$ such that $T_{L 0} \leq T_{L}<\bar{T}_{L}$, because for each $T_{L}$, one of $\left(\theta_{k}, \rho_{k}\right)(k=1,2, \cdots)$ satisfies $\bar{f}^{T_{L}}\left(\theta_{k}, \rho_{k}\right)<0$. This procedure can be applied to the general case, too.

Similarly, starting from $\left(\theta_{1}^{\prime}, \rho_{1}^{\prime}\right):=\left(\theta_{1}, \rho_{1}\right)$, we can compute the smallest negative $\underline{\Delta T_{L}}(\omega)$ such that $f_{\omega}^{T_{L}+\underline{\Delta T}}{ }_{L}(\omega)\left(\theta_{1}^{\prime}, \rho_{1}^{\prime}\right)=0$, if it exists (see Fig. 5) - if no such $\Delta T_{L}(\omega)$ exists, then define $\Delta T_{L}(\omega)=-\infty$. Defining

$$
T_{L 1}^{\prime}=T_{L 0}+\sup _{\omega \in[0, \infty]} \Delta T_{L}(\omega)
$$

we obtain $T_{L 1}^{\prime}=-1.95565<0$. Hence, we can see that stability is assured also for any $T_{L}$ such that $0 \leq T_{L} \leq T_{L 0}$. In the general case, if $T_{L 1}^{\prime}>0$, then we optimize $\bar{f}^{T_{L 1}^{\prime}}(\theta, \rho)$. We would generally obtain $\bar{f}^{T}{ }_{L 1}^{\prime}\left(\theta_{2}^{\prime}, \rho_{2}^{\prime}\right)<0$ for some $\left(\theta_{2}^{\prime}, \rho_{2}^{\prime}\right)$, and then we would obtain $T_{L 2}^{\prime}\left(<T_{L 1}^{\prime}\right)$ using the same procedure. Repeating the procedure, we can obtain the nonincreasing sequence $\left\{T_{L 0}, T_{L 1}^{\prime}, T_{L 2}^{\prime}, \cdots\right\}$, which converges to $\underline{T}_{L}$ (needless to say, if $T_{L k}^{\prime}<0$ for some $k$, we can stop the iteration and set $\underline{T}_{L}=0$ ). This way, we can obtain the lower bound $\underline{T}_{L}$ of $T_{L}$ for which stability can be assured.

\section{CONCLUSION}

In this paper, we studied the input-output stability problem of a class of nonlinear feedback systems and gave a stability criterion with two free parameters. It includes the circle criterion and the Popov criterion as special cases and is most useful in such a practical situation where the nonlinearity in the system is almost time-invariant and memoryless but with slight time-variations and dynamics. It is also shown that the existence of suitable free parameters that fulfill the stability condition can be checked readily by a reduction to a convex problem in the frequency domain. With this convexity approach, we can compute exactly the upper bound for the size of the sector nonlinearity for which we can assure stability. Furthermore, we can also compute the "pure-delay margin" of the system.

\section{APPENDIX \\ Proof of the Main Result}

To prove Theorem 1, note in view of Fig. 2 that $\Sigma_{a}$ (Fig. 1) can be rearranged, using the same $\theta$ as in Theorem 1 , into $\Sigma_{b}$ shown in Fig. 6, where $M_{\theta}$ denotes the multiplier with transfer function

$$
\hat{M}_{\theta}(s)=1+\theta s .
$$

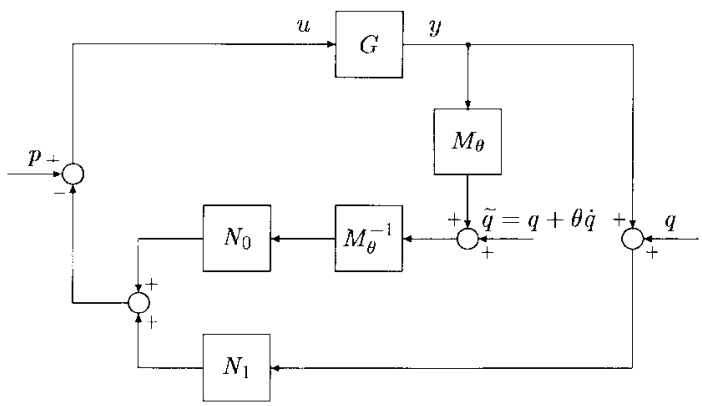

Fig. 6. Introduction of a multiplier (the case of nonnegative $\theta)-\Sigma_{b}$.

We can prove Theorem 1 based on this block diagram. (Here, we confine ourselves to the case of nonnegative $\theta$. If $\theta<0$, we need to rearrange Fig. 1 into Fig. 7 and employ slightly modified arguments, but the details are omitted.)

It is easy to see that $\Sigma_{a}$ is pseudo $L_{2}$-stable if $\Sigma_{b}$ is $L_{2}$-stable for the inputs $p, q$, and $\tilde{q}=q+\theta \dot{q}$. Now, since the nonlinear part $N_{0} M_{\theta}^{-1}$ again belongs to the sector $[0, b]$ in the sense of Definition 2 (see [6]), it can be regarded as the parallel connection of the linear gain $b / 2$ and a new nonlinearity $N_{2}$ belonging to the sector $[-b / 2, b / 2]$. Then, further rearranging the block diagram of $\Sigma_{b}$ (Fig. 6) using these two new elements instead of $N_{0} M_{\theta}^{-1}$, we can obtain the feedback system $\Sigma_{c}$ shown in Fig. 8. Furthermore, it is easy to see that $\Sigma_{b}$ is $L_{2}$-stable for $p, q$, and $\tilde{q}$ if and only if $\Sigma_{c}$ is for the inputs $v=p-b \tilde{q} / 2$ and $w=[q, \tilde{q}]^{T}$.

Now, we can apply the small-gain theorem to $\Sigma_{c}$ if the linear system $G_{m}$ is $L_{2}$-stable. We first show that if (12) is satisfied, then the small-gain condition holds for the feedback system $\Sigma_{c}$; that $G_{m}$ is in fact $L_{2}$-stable if (12) holds will be confirmed later.

Instead of dealing with $\Sigma_{c}$ directly, however, we further apply a loop transformation and introduce a nonzero scaling parameter. To make the arguments transparent, let us introduce

$$
\eta:=\varepsilon^{1 / 2}, \quad \beta:=b^{1 / 2} .
$$

Since $N_{1}$ belongs to the sector $[-\varepsilon, \varepsilon]=\left[-\eta^{2}, \eta^{2}\right]$, and $N_{2}$ belongs to the sector $[-b / 2, b / 2]=\left[-\beta^{2} / 2, \beta^{2} / 2\right]$, the system $\Sigma_{c}$ is $L_{2}$ stable if the system $\Sigma_{d}$ shown in Fig. 9 is $L_{2}$-stable for any $N_{1 s}$ belonging to the sector $[-\eta, \eta]$ and any $N_{2 s}$ belonging to the sector $[-\beta \xi / 2, \beta \xi / 2]$, where $\xi(\neq 0)$ is the scaling parameter. Therefore, by applying the small-gain theorem to $\Sigma_{d}$, we can conclude that the system $\Sigma_{c}$ is $L_{2}$-stable (and hence the system $\Sigma_{a}$ is pseudo $L_{2}$-stable) if

$$
\left\|G_{s}\right\|<\left\|N_{s}\right\|^{-1}
$$

Now, we have the following lemma.

Lemma 1: $\left\|N_{s}\right\|^{2} \leq \varepsilon+b \xi^{2} / 4$.

Proof: Denote, with abuse of notation, the input and output of $N_{s}$ by $u=\left[u_{1}, u_{2}\right]^{T}$ and $y$, respectively. Then, we have $y=N_{1 s} u_{1}+N_{2 s} u_{2}$, so that

$$
\begin{aligned}
\|y\|^{2} & \leq\left(\left\|N_{1 s}\right\| \cdot\left\|u_{1}\right\|+\left\|N_{2 s}\right\| \cdot\left\|u_{2}\right\|\right)^{2} \\
& \leq\left(\left\|N_{1 s}\right\|^{2}+\left\|N_{2 s}\right\|^{2}\right)\left(\left\|u_{1}\right\|^{2}+\left\|u_{2}\right\|^{2}\right) \\
& \leq\left(\eta^{2}+\beta^{2} \xi^{2} / 4\right)\|u\|^{2} \\
& =\left(\varepsilon+b \xi^{2} / 4\right)\|u\|^{2} .
\end{aligned}
$$




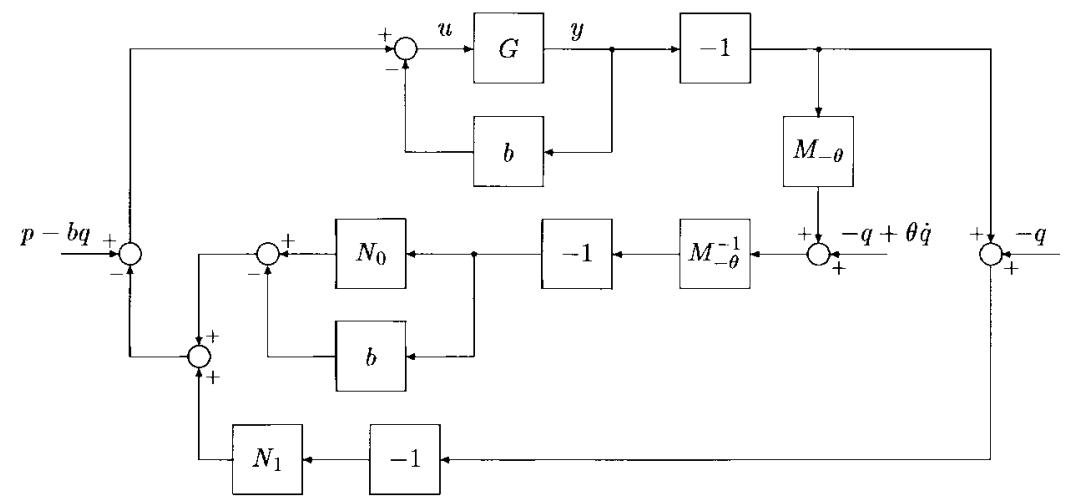

Fig. 7. Introduction of a multiplier (the case of negative $\theta$ ).

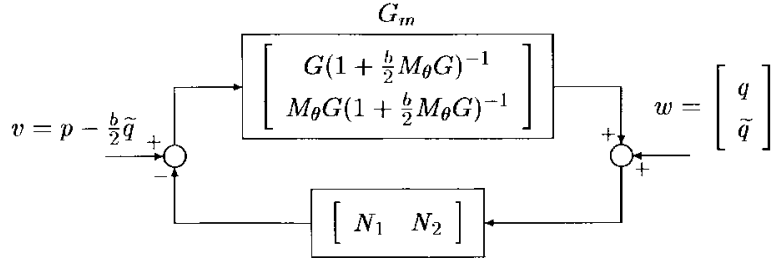

Fig. 8. Equivalent transformation of Fig. $6-\Sigma_{c}$.

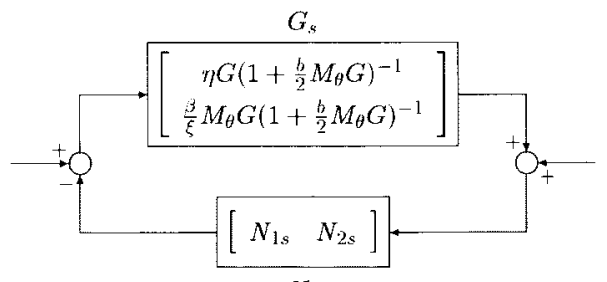

$N_{s}$

Fig. 9. Introduction of scaling into Fig. $8-\Sigma_{d}$.

By the above lemma and (23), together with $\left\|G_{s}\right\|=$ $\sup _{\omega}\left\|\hat{G}_{s}(j \omega)\right\|$, we have only to show that, for $\xi:=(2 \rho)^{1 / 2}$, the condition (12) is equivalent to

$$
\begin{aligned}
& \sup _{\omega \in[0, \infty]}\left\|\hat{G}_{s}(j \omega)\right\|^{2} \\
& =\sup _{\omega \in[0, \infty]}\left(\left|\eta \hat{G}(j \omega)\left(1+\frac{b}{2} \hat{M}_{\theta}(j \omega) \hat{G}(j \omega)\right)^{-1}\right|^{2}\right. \\
& \left.\quad+\left|\frac{\beta}{\xi} \hat{M}_{\theta}(j \omega) \hat{G}(j \omega)\left(1+\frac{b}{2} \hat{M}_{\theta}(j \omega) \hat{G}(j \omega)\right)^{-1}\right|^{2}\right) \\
& \quad<\left(\varepsilon+b \xi^{2} / 4\right)^{-1} .
\end{aligned}
$$

Recalling (22), this can be checked by direct computations.

Finally, the proof becomes complete by showing that $G_{s}$ is $L_{2}$ stable if the condition (12) is satisfied. To prove this, we have only to show that the second subsystem of $G_{s}$, namely $M_{\theta} G(1+$ $\left.b M_{\theta} G / 2\right)^{-1}$, is $L_{2}$-stable [since $M_{\theta}^{-1}$ is $L_{2}$-stable by (21)]. Now, since the condition (12) implies (14) and since $M_{\theta} G$ is $L_{2}$-stable by the assumption $g, \dot{g} \in \mathcal{A}$, it follows from (a version of) the passivity theorem [5] that $M_{\theta} G\left(1+b M_{\theta} G / 2\right)^{-1}$ is $L_{2}$-stable. This completes the proof.

\section{REFERENCES}

[1] S. P. Boyd and C. H. Barratt, Linear Controller Design: Limits of Performance. Englewood Cliffs, NJ: Prentice-Hall, 1991.

[2] C. A. Desoer and M. Vidyasagar, Feedback Systems: Input-Output Properties. New York: Academic, 1975.

[3] E. Furutani, M. Saeki, and M. Araki, "Shifted Popov criterion and stability analysis of fuzzy control systems," in Proc. 31st Conf. Decision and Control, Tucson, AZ, 1992, pp. 2790-2795.

[4] __ "Shifted Popov criterion," Trans. Inst. Syst., Contr. Inform. Eng., vol. 7, no. 7, pp. 247-254, 1994 (in Japanese).

[5] M. Vidyasagar, Nonlinear Systems Analysis, 2nd ed. Englewood Cliffs, NJ: Prentice-Hall, 1993.

[6] G. Zames, "On the input-output stability of time-varying nonlinear feedback systems-Part II: Conditions involving circles in the frequency plane and sector nonlinearities," IEEE Trans. Automat. Contr., vol. AC-11, pp. 465-476, Mar. 1966.

[7] K. Zhou, J. C. Doyle, and K. Glover, Robust and Optimal Control. Englewood Cliffs, NJ: Prentice-Hall, 1996. 